\title{
Design of hybrid wireless sensor network to monitor bioelectric signals focused on the study of epilepsy
}

\author{
Sergio Martínez ${ }^{1}$, Mario Rivero ${ }^{2}$, and Laura Garay ${ }^{3}$ \\ 1 SEPI-ESCOM IPN, Av. Juan de Dios Bátiz Col. Lindavista. Del. Gustavo A. \\ Madero, C.P. 07738 México \\ smartinez@ipn.mx \\ 2 Communication Networks Laboratory, CIC-IPN, Av. Juan de Dios Bátiz, Esq. \\ Miguel Othón de Mendizábal S/N, Nueva Industrial Vallejo, C.P. 07738 México \\ mriveroa@ipn.mx \\ 3 SEPI-UPIITA IPN, Av. Instituto Politécnico Nacional 2580, Barrio La Laguna \\ Ticomán, Gustavo A. Madero, C.P. 07738 México \\ lgaray@ipn.mx
}

\begin{abstract}
Epilepsy is considered a disorder in which a person has certain episodes of disturbed brain activity. There are different studies that allow monitoring physiological signals associated with a possible epileptic episode. However, these require wired connections and therefore monitoring is restricted to factors such as limited physical movements and loss of information by disconnecting sensors. Hence, there is not a system to monitor patients in a mobile (ambulatory) and wireless fashion. This paper describe a design of a wireless sensor network for a body area network $(B A N)$ for continuous monitoring (electroencephalogram $[E E G]$, electrocardiogram $[E C G]$ ) and events monitoring (electrogastrogram $[E G G])$ defined by the expert. The system is based on a TDMA protocol that allows sensor nodes to continuously transmit their information as well as the event nodes access to the network when needed. The system is mathematically studied and numerical results are verified through discrete event simulations.
\end{abstract}

Keywords: Wireless Sensor Network $(W S N)$, Time Division Multiple Access $(T D M A)$, Body Area Network $(B A N)$, Electroencephalography $(E E G)$, Electrocardiography $(E C G)$, Electrogastrography $(E G G)$ and Epilepsy

\section{Introduction}

Epilepsy is one of the most common serious brain diseases. It is a chronic disease with complex side effects on professional, occupational, psychological, social and economical implications [1]. However, in a large number of conditions is not possible to make a diagnosis of epilepsy syndrome. In general, there are symptoms of epilepsy crisis in different body parts; studies carried out today require the 
patient to attend a hospital or medical cabin in order to be monitored using a wired system. As such, patients are greaty restricted during medical analyses.

Addressing the above problematic, an alternative solution is proposed for the evaluation of different studies, including but not restricted to, epilepsy . Medical examinations like electroencephalogram $(E E G)$, electrocardiogram $(E C G)$ and electrogastrogram $(E G G)$ can be monitored using a wireless system, eliminating any restrictions on medical instrumentation wiring. Specifically, a Body Area Network $(B A N)$ through the use of a wireless sensor network (WSN) is proposed for such task. Furthermore, this article develops the design of a hybrid wireless sensor network for continuous monitoring signals of $E E G$ and $E C G$, and event detection driven for $E G G$ signals is evaluated. By this hybrid approach, the system ensures the operation efficiency in terms of channel occupancy and packet loss. A mathematical model and discrete event simulations are performed in order to calculate the system occupancy.

In the literature, there are different $W S N_{\mathrm{s}}$ deployed for $B A N \mathrm{~s}$. However, most studies are focused on other types of measurements, for example, in [46] or [7] where the monitoring of bioelectrical signals are mainly for e-health applications. Nowadays on the market there are non-invasive sensors, conduct to monitoring bioelectric signal and recording anomalies indicating bearer studies and monitoring. Both works, the developed on this article and the market, are made in order to indicate a simple and transparent system to the user, monitoring their respective body functions in different parts and can take the necessary measures to stabilize homeostasis. Brand new sensors like Medtronic or IntraMed have this kind of functionality.

The WSN is based on a TDMA scheme where the first time slots are assigned to the continuous monitoring data transmission while leaving the rest of time slots available for the event-driven detections. By doing this, a collision-free system is design in such a way as eliminating any information loss.

\section{System Model}

In this section, the main assumptions and the protocol operation are explained in detailed. wireless wensor network are composed of a set of autonomous devices (sensor nodes) that are capable of sensing, communicate and operate in a distributed fashion. Nodes are typically randomly scattered in a specified area [2]. In contrast, $W S N \mathrm{~s}$ applied at $B A N \mathrm{~s}$ are placed in a relatively small area. Hence, nodes are rather close to each other. In fact, transmission radius coverage is confined to no more than 2 or 3 meter distances. Also, the energy emissions of such technologies are very low, which contributes to the long life of the system [3]. Transmissions are made within, around or on the human body.

In the particular case of medical measurements for $E E G, E G G$ and $E C G$ analyzes, i.e., for $B A N \mathrm{~s}$ that can be constantly checked by the medical staff, it is also possible to know the exact number of nodes that compose the network (nodes in operating conditions). As such, it is available to replace damaged nodes or even to charge all the node's batteries before operation. Hence, a TDMA 
protocol is proposed because it is a collision-free protocol. As such, there is no energy wastage due to idle listening, collisions or overhearing as in the case of random access protocols, typical in $W S N \mathrm{~s}$. Indeed, in the specific types of $B A N \mathrm{~s}$ considered in this work, it is possible to pre-assign a time slot for each specific node.

In the Fig. 1, it is illustrated the basic operation of the system. It can be seen that in a system with 26 nodes, the TDMA system consists of 26 time slots. Note that in the first part of the TDMA frame, nodes with continuous monitoring tasks are assigned to a particular time slot, which is always occupied with such transmissions. However, the second part of the frame is composed of the event driven transmissions. In this case, we assume a system where $N_{c}$ nodes are deployed for continuous monitoring tasks while $N_{e}$ nodes are focused on event driven reporting. As such, the frame is composed by $N_{c}+N_{e}$ time slots.

It is important to notice that nodes monitoring the $E G G$ signals, are not required to constantly transmit all the sensed information. In particular, the medical staff is only interested on receiving information when a certain predefined event occurs. For instance, when signals measured from the patient are outside the normal operation range. As such, nodes do not constantly transmit in tis part of the frame. However, the system guarantees the successful transmission of these events. In the first part of the frame, the channel occupation is always $100 \%$. In the second part of the frame, the occupancy greatly depends on the rate at which events occur, i.e., how often a patient experiences out of range activity in the heart.

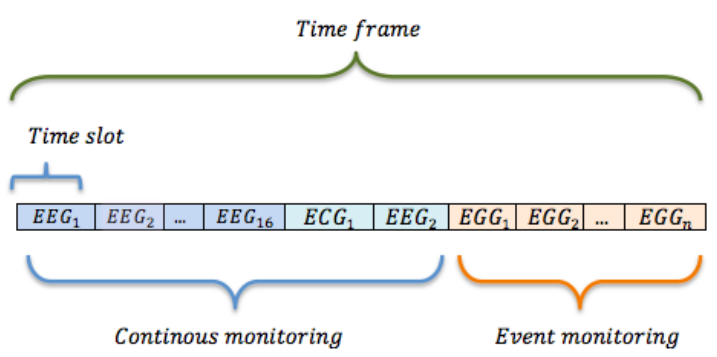

Fig. 1. TDMA-based system

\section{Mathematical Model}

The mathematical model presented in this section is mainly focused on the channel occupation in the event reporting phase. To this end, an event arrival rate of $\lambda_{e}$ events per second is assumed. Events in this context refers to the 
readings of the $E G G$ signals that falls outside the normal range for the particular patient. Note that the system can be further developed in order to consider multiple events. Indeed, the proposed $B A N$ can easily adapt to multiple types of biological sensors with very different event characteristics. It is also assumed that events arrive according to a Poisson process, (the time between events are a random variable with negative exponential distribution with mean $1 / \lambda_{e}$ seconds). Again, the event distribution can change for different patients and biological signals. However that does not impact the system operation since the network has always available resurfaces for the nodes in order to transmit their information. The occupancy calculated in this work can be quiet different in case that events follow a different distribution.

In the proposed model, it is consider the case where sensor nodes do not detect the event, (it does not consider a perfect sensing system). The rationale behind this is that, nodes are placed in the clothes or an elastic band around the interest area. Hence, in an ambulatory device, it is possible for nodes to move due to the patients position or movement. So, whenever the event occurs, the sensor nodes will not detect the appropriate signal. We model this by introducing the probability, $\rho$ that, given that an event occur, a particular sensor node detect it and transmit the associated information. On the other hand, a node does not detect the event with probability $1-\rho$ and hence, it does not transmit packets in the appropriate time slot. From the previous description, the channel occupancy in the event report in phase can be calculated as follows:

$$
O_{e}=\frac{\lambda_{e} \sum_{i=0}^{n} i\left(\begin{array}{c}
N_{e} \\
i
\end{array}\right) \rho^{i}(1-\rho)^{\left(N_{e}-1\right)}}{N_{e}}
$$

Occuppancy refers to the number of $N_{e}$ slots per frame being used to transmit information.

\subsection{Discrete event simulation}

Discrete events simulations are implemented in order to verify the mathematical analysis. It is considered that 18 nodes are deployed for continuous monitoring $\left(N_{c}=18\right)$ where 16 channels are on the $E E G$ and the remaining 2 are for $E C G$. Also, the event transmission nodes $\left(N_{e}\right)$ are subject to variation starting with at least 2 sensors for electrogastrography up to 10. It is also considered that the probability of detecting the event whenever it occurs, $\rho$, can vary in the range of 0.2 to 0.7 .

\section{Numerical Results}

It is consider three different values of the event arrival rate, $\lambda_{e}$ and the performance evaluation of the system. we present numerical results for different values of $\rho$ and $N_{e}$. In all cases, $N_{c}=18$. Fig. 2 presents results for a value of $\lambda_{e}=0.3$.

On each occasion that the ocuppancy term is used, it is referring to the portion of time slots that are being used. It can be seen that the values obtained in 


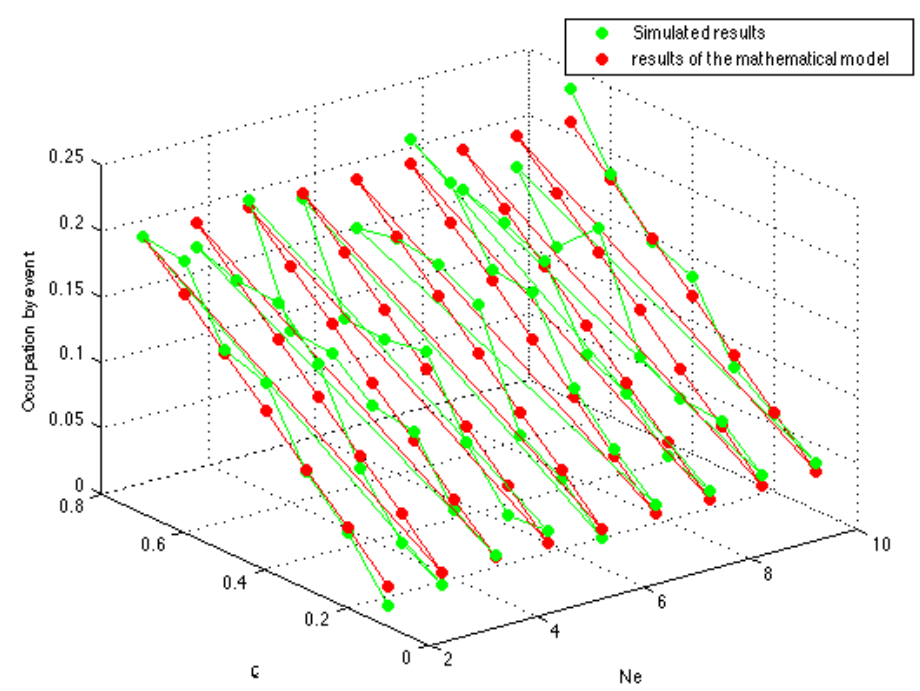

Fig. 2. Occupation per event $\lambda_{e}=0.3$ and $N_{c}=18$

the simulation are very similar to the mathematical model. Occupancy decreases as the value of $\rho$ decreases. Indeed, as the probability that nodes detect the event is low, there are just a few transmissions, leaving many time slots empty. This can correspond to the case of the patient in movement, walking or jogging for instance. In this sense, it is advisable to use many redundant nodes in order to capture readings in case of an event occurrence.

Fig. 3 and 4 present results for $\lambda_{e}=0.5$ and $\lambda_{e}=0.7$ respectively. This case corresponds to a system with more heart activity. Note that occupancy increases compared to the previous figure.

\section{Conclusion}

Considering the EEG, ECG and $E G G$ studies by a separate set of interconnected nodes, a design was developed for a hybrid $W S N$ using a TDMA-based protocol. A mathematical model and the respective discrete event simulations for comparison of results was performed. Performance evaluation is made in terms of the channel occupancy validating the mathematical model. It is considered that based on the results, the maximum efficiency is obtained for a high rate of arrivals and to a high probability of transmission nodes per event. However, channel occupancy is never higher that $50 \%$ in the considered environments.

It is important to notice that even for high arrival rates and considering that nodes detect most events, for instance $\rho=0.7$, the channel occupancy is 


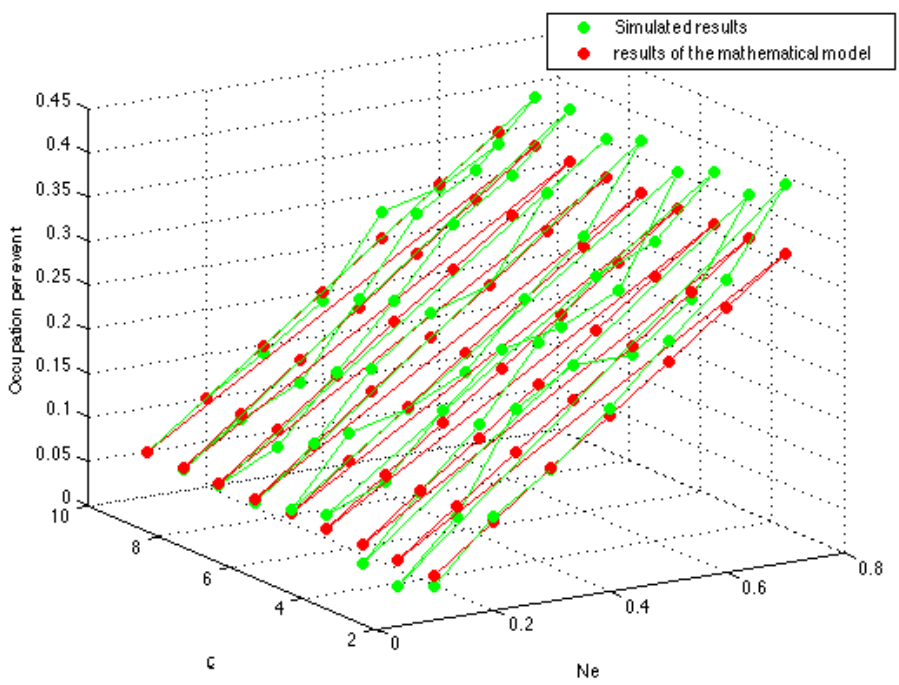

Fig. 3. Occupation per event $\lambda_{e}=0.5$ and $N_{c}=18$

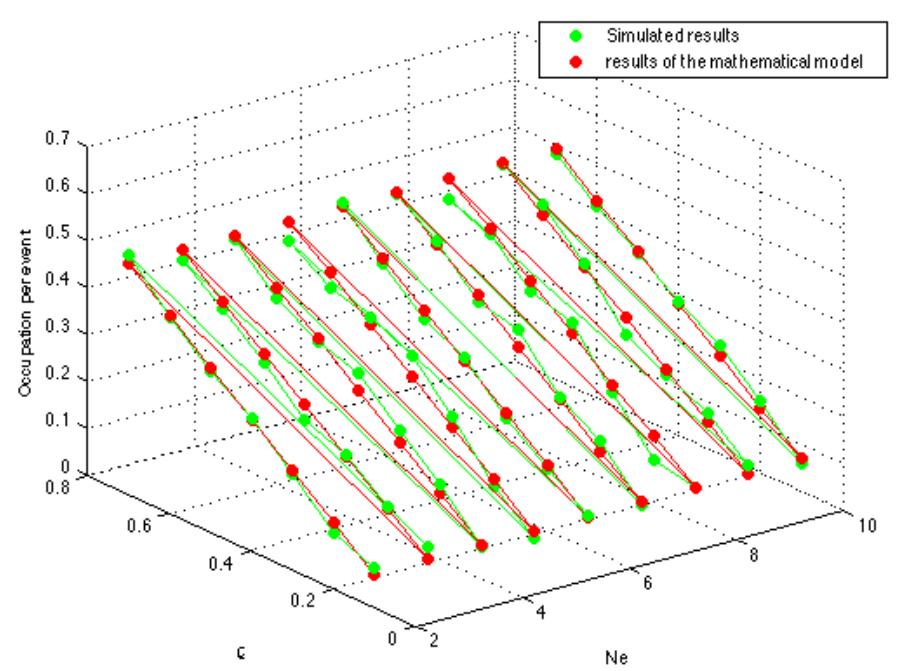

Fig. 4. Occupation per event $\lambda_{e}=0.7$ and $N_{c}=18$ 
rather low. This is because when no event occurs, all the times slots dedicated to event reporting are empty. This suggests that a more efficient system can be implemented that takes advantage of this fact.

\section{References}

1. Hernndez, A.; vila, L.; Arch, E.; Bueno, A.; Espinoza, G.; Alfaro, A.: La epilepsia como un problema de discapacidad. Disability Research, 2, 122-130 (2013)

2. Dargie, W.; Poellabauer, C.: Fundamentals of Wireless Sensor Network. John Wiley \& sons Ltd (2010)

3. Enderle, J.; Blanchard, S.; Bronzino, J.: Introduction to biomedical engineering. Elsevier academic press (2004)

4. Chung, W. Y.; Lee, Y. D.; Jung, S. J.: A Wireless Sensor Network Compatible Wearable U-healthcare Monitoring System Using Integrated ECG, Accelerometer and SpO2. Institute of Electrical and Electronics Engineers, 8, 1529-1532 (2008).

5. Castillejo, P.; Martnez, J. F.; Rodrguez, J. M.: Integration of wearable Devices in a wireless sensor network for an e-health application, Institute of Electrical and Electronics Engineers, 13, 38-49 (2013)

6. Phunchongharn, P.; Hossain, E.; Camorlinga, S.: A cognitive radio system for Ehealt applications in a hospital environment, Institute of Electrical and Electronics Engineers, 10, 20-28 (2010)

7. Sivaraman, V.; Grover, S.; Kurusingal, A.; Dhamdhere, A.; Burdett, A.: Experimental study of mobility in the soccer field with application to real-time athlete monitoring, Institute of Electrical and Electronics Engineers, 6, 337-345 (2010) 